Keio J. Med. 24: 399-405, 1975

\title{
LYMPHOCYTE MEDIATED CYTOTOXICITY IN HUMAN RENAL ALLOTRANSPLANTATION
}

\author{
MAKOTO HATA* and HIROSHI NAKAMURA** \\ * The Department of Urology, School of Medicine, Keio University, Tokyo \\ ** Department of Urology, Kitasato Institute Hospital, Shirogane, Tokyo
}

\begin{abstract}
The purpose of this study is to assess the correlation between lymphocyte mediated cytotoxicity and laboratory data in six patients with renal allografts, four of whom underwent thymectomy also. Cell mediated cytotoxicity against Hela cells as target is assayed after preparation of lymphocytes from the peripheral blood by Ficoll-hypaque method. Tests are conducted in microtest tissue culture plates according to the procedure described by Takasugi and Klein in 1970. Results are expressed by mean reduction per titer. In the group of patients who had run uneventful course after renal allotransplantation with standard immunosuppression, lymphocytes failed to demonstrate a potent cytotoxic effect, meanwhile survival of target cells was noticoably reduced by lymphocytes from patients who were suffering from any sorts of disorder such as acute and chronic rejection, toxic hepatitis, post surgical risk and so forth. The direction of reactivity that might be suppressed in the transplant recipients with thymectomy as adjunctive, gives rise to the possibility that this lymphocyte mediated cytotoxicity test reflects at least partly T-cell derived immunity.
\end{abstract}

In 1973 Takasugi et al. observed the reactivity of lymphocytes from cancer patients on the same histological type of cultured tumor cells in an attempt to detect the specific cell mediated immunity to human cancer according to histological type. ${ }^{2}$ The weaker effect by normal persons is in agreement with the general understanding that individual who have not had cancer are usually nonreactive in cell-mediated cytotoxicity test against cultured tumor cells, whereas lymphocytes from patients with same histological type of cancer may remarkably decrease target cell survival. Contrary to this expectation, from an extensive series of tests they have shown that lymphocytes from normal persons are as reactive as lymphocytes from cancer patients, if not more active than.

By the way what will happen if we compare the activity of lymphocytes from normal healthy subjects on the established cell line as target, with that of patients with different histological type of cancer. Lymphocyte mediated cytotoxicity 
against Hela cells as target was assayed in patients with advanced bladder carcinoma and was shown to be diminished, compared with that in normal healthy controls (unpublished data). In view of the fact that lower activity of cell mediated immunity determined by non-specific systems such as PHA response and DNCB skin test is generally accepted in the patients with advanced carcinoma, it is suggested that this lymphocyte mediated cytotoxicity test may also reflect the non-specific immune response.

The purpose of this study is to determine the non-specific immune capacity by means of lymphocyte mediated cytotoxicity against Hela cells as target in patients with renal allografts and its relationship with their clinical data is reported herein.

\section{PATIENT PROTOCOL}

From 1968 to date we have accumulated 10 patients who were with end stage renal failure and treated by living related renal transplantation. Four of ten are living as of January 1,1975 . The longest one survived 2.177 days. Of ten patients six with the star on the left of their initial were submitted to this study (Table 1). Four of six candidates, underwent thymectomy as well before or after renal transplantation.

Table 1

Transplant recipients

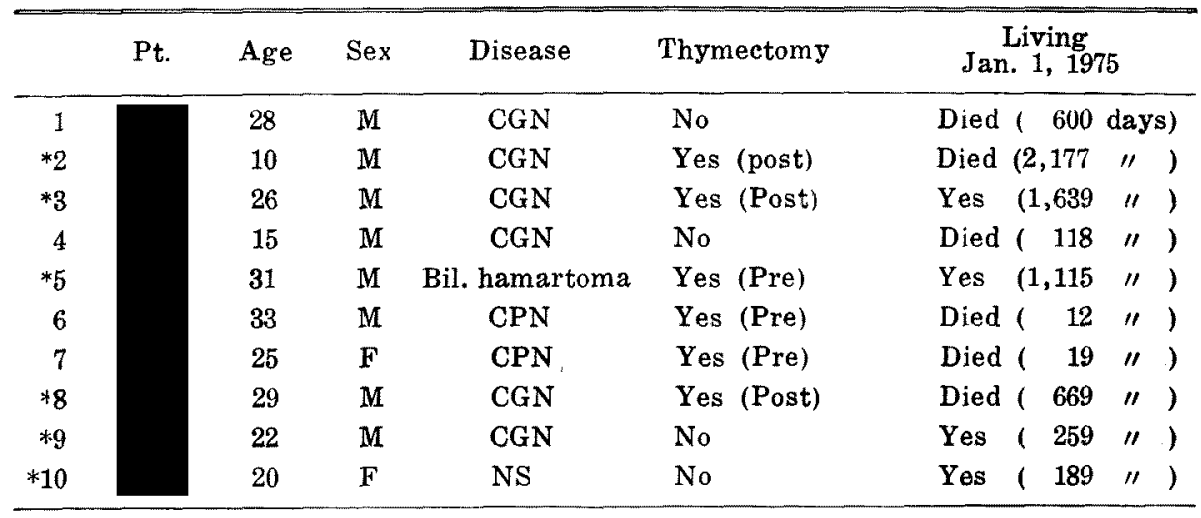

The lymphocyte mediated cytotoxicity test was performed by the method of Takasugi \& Klein in $1970 .^{3}$ Tests were conducted in microtest tissue culture 
plates, composed of 60 wells in each. Each well has capacity of $20 \mu \mathrm{l}$. A few hours before the test, Hela cells as target, were harvested by treatment with $0.25 \%$ trypsin. After washing and resuspending in fresh medium, the cells were counted and diluted to deliver 500 cells in $10 \mu \mathrm{l}$ and then plated into each well of the plastic tray. All lymphocytes as effector were separated from the peripheral venous blood of the patients tested according to the Ficoll-hypaque method $^{1}$ and also washed three times by centrifugation and cell numbers were adjusted to desired levels. In addition to the assessment of cytotoxic activity for each cell suspension, determination of its relative strength was made by cell titration. Original counts were adjusted to deliver 25,000 cells in $10 \mu \mathrm{l}$, representing effector to target cell ratio of $50: 1$, and serial 2 -fold dilutions were made. Then effector cells were overlaid on the target cells at each levels. The plates were incubated at $37^{\circ} \mathrm{C}$ in an atmosphere of $5 \% \mathrm{CO}_{2}$ for 2 days. The test was terminated by washing each well twice with normal saline solution to preserve only viable cells. Living cells attached to the floor of the well were fixed for $30 \mathrm{~min}$. in methanol and were stained with Giemsa.

The number of target cells remaining after fixing and staining was used to calculate the percentage of reduction, by the following formula:

$$
\frac{(\text { No. of cells in target cell control) }-(\text { No. of cells in test) }}{\text { No. of cells in target cell control }} \times 100
$$

Results were expressed by mean reduction per titer (MRT). The percentage of reduction was determined at the 4 effector to target cell ratios and was designated $a, b, c$, and $d$. Then the MRT score was calculated as following:

MRT score $=(a+b+c+d) / 4$

\section{RESULTS}

The MRT score and other clinical data at the time of test, including peripheral lymphocyte counts, serum creatinine levels, immunosuppressive agents, results of DNCB skin test and clinical pictures are summarized in Table 2. The MRT score obtained from the normal healthy population ranged from 43 to $54 \%$. When the No. 1 patient was tested his renal graft was in chronic rejection with serum creatinine level of $3.5 \mathrm{mg} / \mathrm{dl}$ and his lymphocytes highly reduced target cell survival. Shortly after the time of this test he was succumbed to the generalized bacterial infection at the 2,177th day post operatively. No. 2 patient is one of the long term survivors. His lymphocytes tested when he was in good clinical course, demonstrated the diminished cytotoxicity against the target cells. No. 3 patient is another long term survivor and his lymphocytes also showed low 
Table 2

Comparison of MRT with other clinical data

\begin{tabular}{|c|c|c|c|c|c|c|c|}
\hline & \multirow[b]{2}{*}{ PLC } & \multirow[b]{2}{*}{$\mathrm{SC}$} & \multicolumn{3}{|c|}{ Immunosuppression } & \multirow[b]{2}{*}{$\begin{array}{l}\text { Clin. } \\
\text { Diag. }\end{array}$} & \multirow[b]{2}{*}{ MRT } \\
\hline & & & $\begin{array}{l}\text { Azathio. } \\
\text { or Cyclph. } \\
\text { (mg) }\end{array}$ & $\begin{array}{l}\text { Pred. or } \\
\text { B-Meth } \\
\text { (mg) }\end{array}$ & DNCB & & \\
\hline 1 & 1,898 & 3.50 & 75 & 7.5 & ${ }_{(++)}^{\text {impaired }}$ & chr. rej. & 50.8 \\
\hline 2 & 3,392 & 1.39 & 125 & 3.0 & ${ }_{(++)}^{\text {impaired }}$ & good & 15.3 \\
\hline \multirow[t]{2}{*}{3} & 1,392 & 1.70 & 150 & 7.0 & \multirow{2}{*}{$\underset{(+)}{\text { impaired }}$} & good & 7.7 \\
\hline & 2,925 & 1.80 & 125 & 8.0 & & good & 28.7 \\
\hline 4 & 190 & 2.63 & 25 & 30.0 & \multirow{4}{*}{$\underset{(+)}{\text { impaired }}$} & chr. rej. & 27.4 \\
\hline \multirow[t]{3}{*}{5} & 1,440 & 1.06 & 175 & $2.0(\beta)$ & & $\operatorname{good}$ & 9.2 \\
\hline & 316 & 1.54 & 175 & $13.0(\beta)$ & & act. rej. & 29.1 \\
\hline & 792 & 1.61 & 175 (C) & $1.5(\beta)$ & & $\begin{array}{l}\text { toxic } \\
\text { hepatitis }\end{array}$ & 67.5 \\
\hline 6 & 1.281 & 1.84 & 75 & $1.5(\beta)$ & anergy & $\begin{array}{l}\text { post } \\
\text { nephrectomy }\end{array}$ & 45.3 \\
\hline
\end{tabular}

MRT score. At the second time of test, however, his lymphocyte response against the target cells was relatively high in spite of no particular change in his clinical picture, although the MRT score was still far under the lower limit of normal persons. No. 4 patient had been suffering from the chronic rejection with slowly increasing level of serum creatinine and had relatively high MRT score one and half months prior to his death at the 669 th day post operatively. Our preliminary study in canine renal allograft indicated that beta-methasone could replace part of prednisolone as immunosuppressive agent and was less toxic (unpublished data). Therefore the following two patients have been maintained with betamethasone instead of prednisolone in its equivalent potency. When No. 5 patient was tested first, he was in uneventful convalescence and the MRT score was as low as that of No. 3 patient. On the recovery from the marginal acute rejection crisis, and when being treated with increased dose of beta-methasone from 2 to $13 \mathrm{mg} /$ day, his lymphocytes reduced noticeably target cell survival. Although he was thought to be completely recovered from acute rejection crisis and had been treated with maintainance dose of immunosuppressive agents when he was tested on the 3rd occasion, his lymphocytes reacted very strongly against the target cells. At this time he was receiving cyclophosphamide instead of azathioprine since his clinical data appeared compatible with toxic hepatitis caused by azathioprine. No. 6 patient was free of complications after transplantation inasmuch as the renal graft function was concerned. Because of the refractory hyper- 
tension, however, she had to have her own kidneys removed 6 months after renal transplantation. Subsequently her lymphocytes were tested, 40 days after the nephrectomy, and showed rather strong cytotoxicity.

DNCB skin tests were performed in 5 of 6 candidates as adjunctive to detect their cellular immunity and were more of less impaired.

Figure 1 depicts the correlation between the MRT score and peripheral lymphocyte count. There did not seem to be any correlation between these two parameters.

Correlation between the MRT score and serum creatinine level was observed in Figure 2. Positive correlation could be not necessarily seen between these two parameters again.

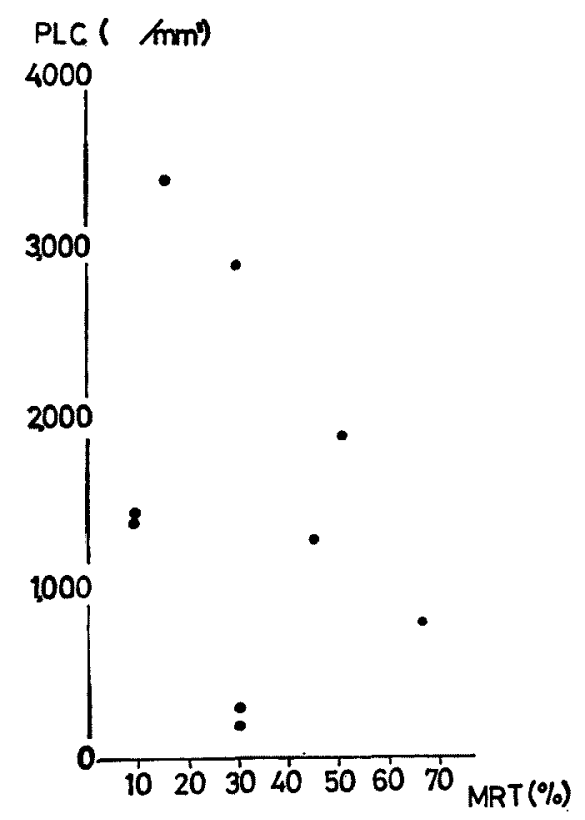

Fig. 1

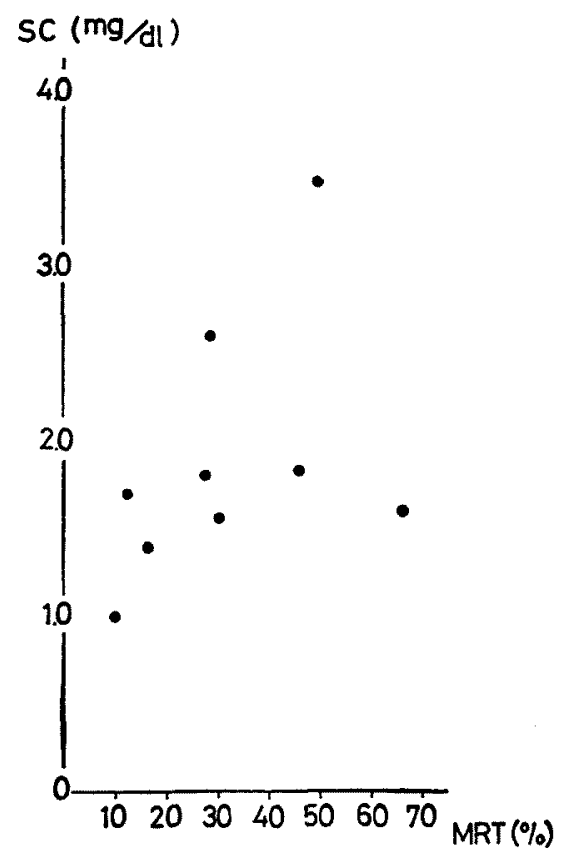

Fig. 2

Fig. 1 Correlation between the mean reduction per tiers and the peripheral lymphocyte counts.

Fig. 2 Correlation between the mean reduction per titers and the levels of serum creatinine.

Each of the MRT score was plotted into two columns regardless to their 
other clinical data (Figure 3). The MRT score from thymectomized patients was plotted on the left column and that from non-thymectomized on the right. Lymphocyte mediated cytotoxicity appears to be influenced by other factors more in the non-thymectomized patients than in the thymectomized patients.

\section{$\operatorname{MRT}(\%)$}



Fig. 3

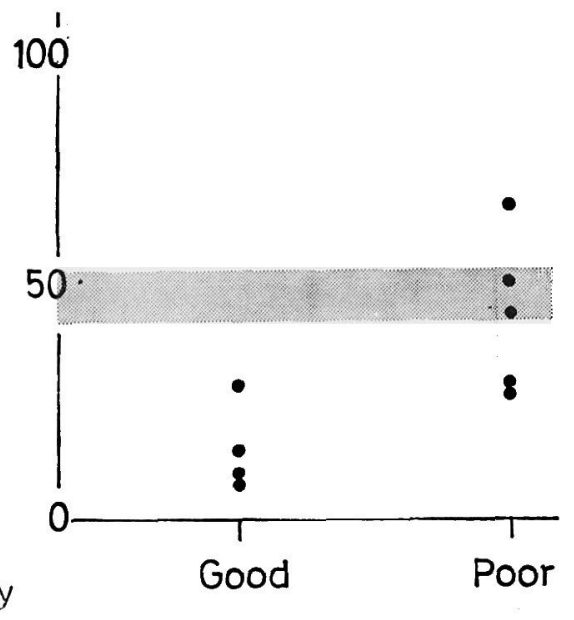

Clinical Course

Fig. 3 Mean reduction per titers of the thymectomized transplant are assigned to the right column. Shadowed area represents the range of mean reduction per titer of the normal healthy persons.

Fig. 4 Mean reduction per titer of the patients tested when they were in good clinical course are assigned to the left column and those of the patients in poor clinical course to the right column. Shadowed area represents the range of mean reduction per titer of the normal healthy persons.

In Figure 4 each of the MRT score was assigned into two groups. In one group lymphocyte mediated cytotoxicity test was carried out when the patients were in good clinical course. In the other group lymphocytes were tested when their clinical picture were designated as poor. Apparently lymphocytes from the patients tested when they were in poor condition reacted more strongly against the target cells. 


\section{DISCUSSION}

Because the number of patients and occasions tested is limited and moreover this system of lymphocyte mediated cytotoxicity test is likely affected by other non-immune factors, only little can be concluded through this study. It is worthwhile, however, to note that in the group of patients who had run uneventful course after renal allotransplantation with standard immunosuppression, lymphocytes failed to demonstrate a potent cytotoxic effect, meanwhile survival of target cells was noticeably reduced by lymphocytes from patients who were suffering from whatever kind it was. Besides, the direction of reactivity that might be suppressed in the transplant recipients with thymectomy as adjunctive, gives rise to the possibility that this system of lymphocyte mediated cytotoxicity test may reflect at least partly the T-cell derived immunity.

\section{SUMMARY}

Reactivity of lymphocytes against the established cell line, Hela cells, as target is observed in six patients who have received living related renal allograft, in an attempt to detect their non-specific immune response. In addition to the standard immunosuppressive agents four of the six patients have been treated by thymectomy as well before or after the renal allotransplantation. Variety of factors influencing upon the results of this lyphocyte mediated cytotoxicity test are discussed.

\section{REFERENCES}

1. Böyum, A.: Isolation of mononuclear cells and granulocytes from human blood. Scand. J. Clin. Lab. Invest. Suppl. 21, 97: 77, 1968

2. Takasugi, M., Mickey, M. R. and Terasaki, P. I.: Reactivity of Iymphocytes from normal persons on cultured tumor cells. Cancer research 33: 2898, 1973

3. Takasugi, M. and Klein, E.: A Microassay for cell-mediated immunity. Transplantation 9: 219, 1970 